\title{
Unitarity Triangle analysis beyond the Standard Model from UTfit
}

Marcella Bona* on behalf of the UIfit Collaboration ${ }^{\dagger}$

Queen Mary University of London

E-mail: m.bonaegmul.ac.uk

The Unitarity Triangle (UT) analysis can be used to constrain the parameter space in possible new physics (NP) scenarios. We present here an update of the UT analysis beyond the Standard Model (SM) by the UTfit collaboration. Assuming NP, all of the available experimental and theoretical information on $\Delta F=2$ processes is combined using a model-independent parametrisation. We determine the allowed NP contributions in the kaon, $D, B_{d}$, and $B_{s}$ sectors and, in various NP scenarios, we translate them into bounds for the NP scale as a function of NP couplings.

38th International Conference on High Energy Physics

3-10 August 2016

Chicago, USA

\footnotetext{
${ }^{*}$ Speaker.

${ }^{\dagger}$ The UIfit Collaboration: Cristiano Alpigiani (University of Washington), Adrian Bevan (Queen Mary University of London), Marcella Bona (Queen Mary University of London), Marco Ciuchini (INFN Sezione di Roma Tre), Denis Derkach (Yandex/Higher School of Economics), Enrico Franco (INFN Sezione di Roma), Vittorio Lubicz (University of Roma Tre), Guido Martinelli (University of Roma La Sapienza), Fabrizio Parodi (University of Genova and INFN), Maurizio Pierini (CERN), Luca Silvestrini (INFN Sezione di Roma), Achille Stocchi (LAL-IN2P3 Orsay), Cecilia Tarantino (University of Roma Tre), Vincenzo Vagnoni (INFN Sezione di Bologna).
} 


\section{Introduction}

Flavor physics represents a powerful tool to test the SM and from the global fit we can extract the most accurate determination of the parameters of the Cabibbo-Kobayashi-Maskawa (CKM) matrix [1,2], as well as the best SM predictions of flavour observables. Improving the accuracy on the CKM parameters is at the heart of many searches for NP, where small NP effects are looked for. The UT analysis performed by the UTfit Collaboration follows the method described in refs. [3, 4] and is updated with the latest determinations of the theoretical inputs and the latest measurements of the experimental observables. The results of the SM analysis can be found in [5]. The complete set of numerical values used as inputs can be found at URL [6] in the Summer 2016 section.

Here we present the results of the full analysis of the UT reinterpreting the experimental observables including possible model-independent NP contributions. The possible NP effects considered in the analysis are those entering neutral meson mixing ( $\Delta F=2$ transitions) and they can be parameterised in a model-independent way with the two following sets of parameters:

$$
\frac{\left\langle B_{q}\left|H_{\mathrm{eff}}^{\mathrm{full}}\right| \bar{B}_{q}\right\rangle}{\left\langle B_{q}\left|H_{\mathrm{eff}}^{\mathrm{SM}}\right| \bar{B}_{q}\right\rangle}=C_{B_{q}} e^{2 i \phi_{B_{q}}}=\left(1+\frac{A_{q}^{\mathrm{NP}}}{A_{q}^{\mathrm{SM}}} e^{2 i\left(\phi_{q}^{\mathrm{NP}}-\phi_{q}^{\mathrm{SM}}\right)}\right)
$$

where in the SM $C_{B_{d, s}}=1$ and $\phi_{B_{d, s}}=0$, or equivalently $A_{q}^{\mathrm{NP}}=0$ and $\phi_{q}^{\mathrm{NP}}=0 . H_{\text {eff }}^{\mathrm{SM}}$ is the $\mathrm{SM}$ $\Delta F=2$ effective Hamiltonian, $H_{\text {eff }}^{\text {full }}$ is its extension in a general NP model, and $q=d$ or $s$.

The following experimental inputs are added to the fit to extract information on the $B_{s}$ system: the semileptonic asymmetry in $B_{d}$ and $B_{s}$ decays, the di-muon charge asymmetry [7], the $B_{s}$ lifetime from flavour-specific final states, and CP-violating phase and the decay-width difference for $B_{s}$ mesons from the time-dependent angular analyses of $B_{s} \rightarrow J / \psi \phi$ decays. The values used as inputs are mostly taken from the Heavy Flavor Averaging Group [8], except for the $B_{S}$ semileptonic asymmetry where an updated average is available in ref. [9].

\section{Result of the model-independent global fit analysis}

Using the above inputs and our Bayesian framework, we perform the full NP analysis and the result of this global fit selects a region of the $(\bar{\rho}, \bar{\eta})$ plane which is consistent with the result of the SM analysis. This can be seen in the $\bar{\rho}-\bar{\eta}$ plane in Fig. 1 . The $\bar{\rho}$ and $\bar{\eta}$ value extracted are $\bar{\rho}=0.150 \pm 0.027$ and $\bar{\eta}=0.363 \pm 0.025$, that can be compared to the SM ones: $\bar{\rho}=0.154 \pm 0.015$ and $\bar{\eta}=0.344 \pm 0.013$ [5]. Simultaneously to the $\bar{\rho}$ and $\bar{\eta}$ parameters, the NP parameters are extracted in the NP global fit: Fig. 2 show the result of the fit in the $\phi_{B_{d}, s}-C_{B_{d}, s}$ planes directing assessing how the model-independent NP parameters are constrained by the current flavour picture. The NP parameters in the $B_{d}$ and $B_{s}$ systems are found in agreement with the SM expectations with values $C_{B_{d}}=1.04 \pm 0.12, \phi_{B_{d}}=(1.8 \pm 1.7)^{\circ}, C_{B_{s}}=1.07 \pm 0.09$ and $\phi_{B_{s}}=(0.1 \pm 1.0)^{\circ}$.

Using the alternative parameterisation $A_{q}^{\mathrm{NP}} / A_{q}^{\mathrm{SM}}$ and $\phi_{q}^{\mathrm{NP}}$, Fig. 3 show the values still available for the NP parameters in the $B$ systems. Currently, the ratio of NP/SM amplitudes needs to be less than $15 \%$ at $68 \%$ probability in both $B_{d}$ and $B_{s}$ mixing (at $95 \%$ probability the ratio increases as $30 \%$ for $B_{d}$ and $25 \%$ for $B_{s}$ ). The good consistency of the SM picture allows for constraining the amount of NP that can still be allowed to contribute, however a $15-25 \%$ effect is still allowed given the current sensitivities. 


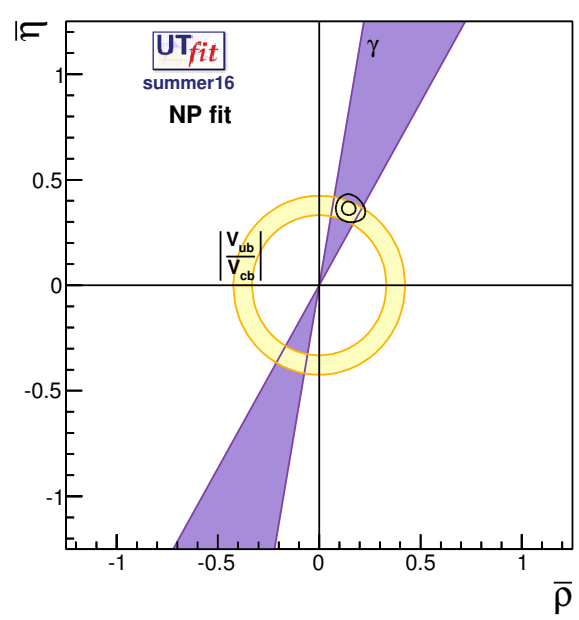

Figure 1: $\bar{\rho}-\bar{\eta}$ plane showing the result of the NP fit. The black contours display the $68 \%$ and $95 \%$ probability regions selected by the given global fit. The $95 \%$ probability regions selected are also shown for those constraints not affected by NP in $\Delta F=2$ transitions, however all the constraints are used in the fit selecting the $\bar{\rho}-\bar{\eta}$ area.
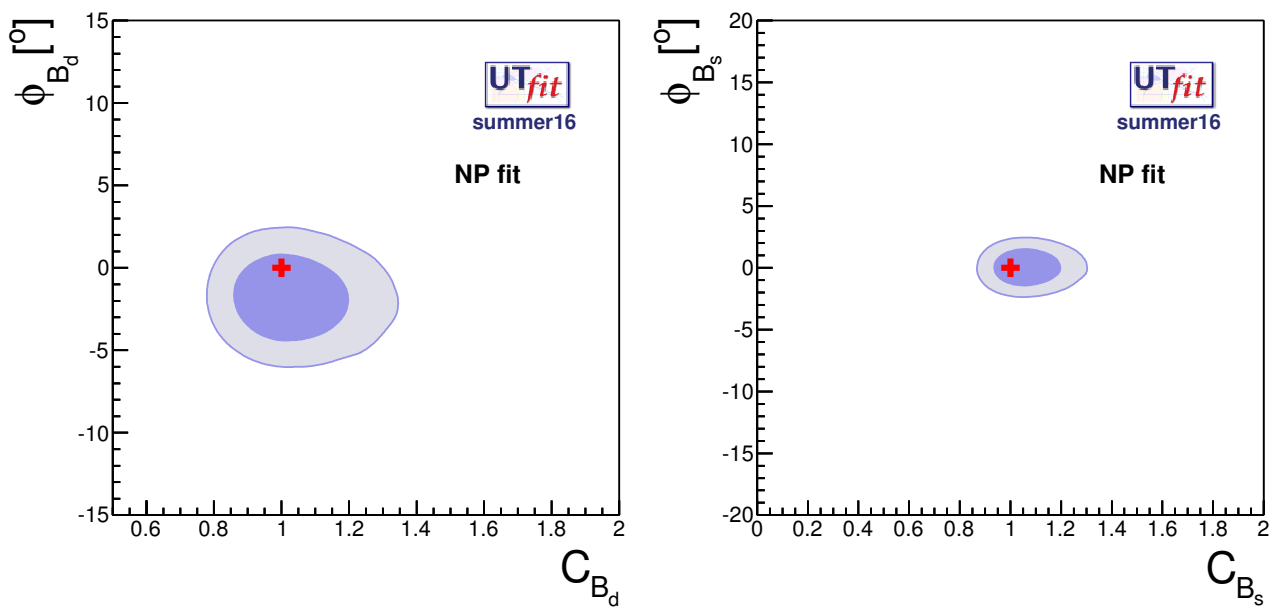

Figure 2: NP parameters in the $B_{d}$ system (left) and the $B_{s}$ system (right). The $68 \%$ (dark) and $95 \%$ (light) probability regions are shown in the $\phi_{B_{d}, s}-C_{B_{d}, s}$ planes as obtained in the NP fit. The red cross represents the SM expectation.

\section{New-physics scale analysis}

We can now consider the most general effective Hamiltonian for $\Delta F=2$ processes $\left(\mathscr{H}_{\text {eff }}^{\Delta F=2}\right)$ in order to translate the current constraints into allowed ranges for the Wilson coefficients of $\mathscr{H}_{\text {eff }}^{\Delta F=2}$. The full procedure and analysis details are given in [10]. These coefficients have the general form

$$
C_{i}(\Lambda)=\frac{F_{i} L_{i}}{\Lambda^{2}}
$$

where $F_{i}$ is a function of the (complex) NP flavour couplings, $L_{i}$ is a loop factor that is present in models with no tree-level Flavour Changing Neutral Currents (FCNC), and $\Lambda$ is the scale of NP, 

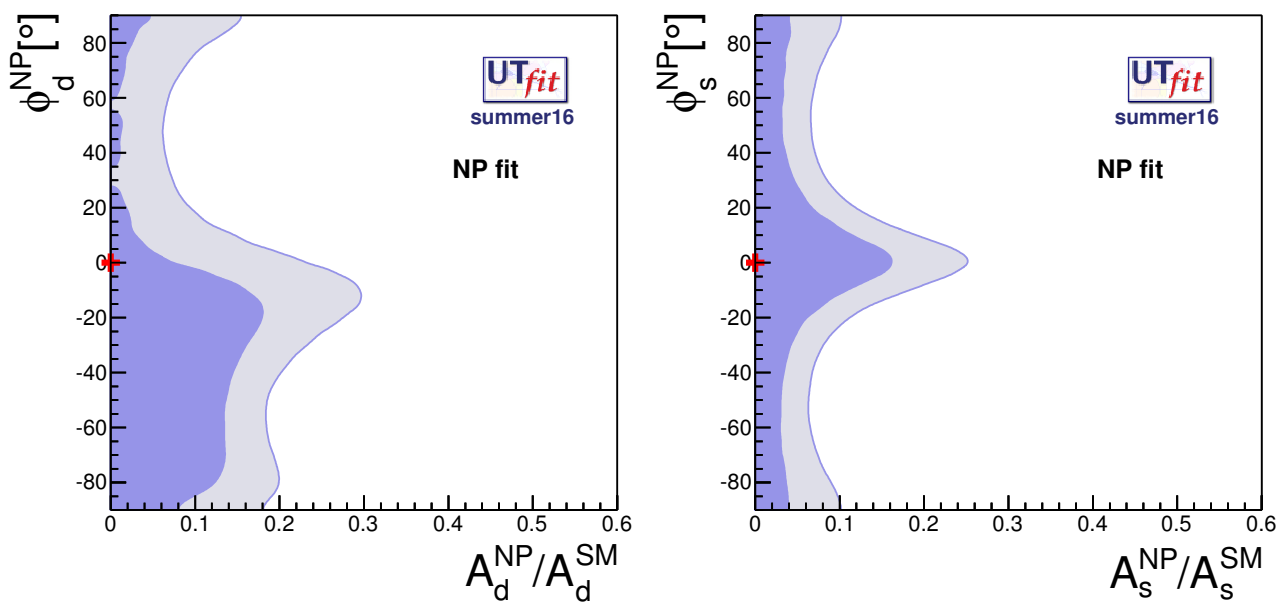

Figure 3: NP parameters in the $B_{d}$ system (left) and $B_{s}$ system (right), where $68 \%$ (dark) and 95\% (light) probability regions are shown in the $A_{q}^{\mathrm{NP}} / A_{q}^{\mathrm{SM}}-\phi_{q}^{\mathrm{NP}}$ planes in the NP fit. The red cross represents the SM expectation.

i.e. the typical mass of the new particles mediating $\Delta F=2$ transitions. For a generic stronglyinteracting theory with arbitrary flavour structure, one expects $F_{i} \sim L_{i} \sim 1$ so that the allowed range for each of the $C_{i}(\Lambda)$ can be immediately translated into a lower bound on $\Lambda$. Specific assumptions on the flavour structure of NP, for example Next-to-Minimal [11] Flavour Violation (NMFV), corresponds to particular choices of the $F_{i}$ functions.

The most general effective Hamiltonians for $\Delta F=2$ processes beyond the SM have the following form, e.g. for $\Delta B=2$ :

$$
\mathscr{H}_{\mathrm{eff}}^{\Delta B=2}=\sum_{i=1}^{5} C_{i} Q_{i}^{b q}+\sum_{i=1}^{3} \tilde{C}_{i} \tilde{Q}_{i}^{b q}
$$

where $q=d(s)$ for $B_{d(s)}-\bar{B}_{d(s)}$ mixing and

$$
\begin{gathered}
Q_{1}^{q_{i} q_{j}}=\bar{q}_{j L}^{\alpha} \gamma_{\mu} q_{i L}^{\alpha} \bar{q}_{j L}^{\beta} \gamma^{\mu} q_{i L}^{\beta}, \\
Q_{2}^{q_{i} q_{j}}=\bar{q}_{j R}^{\alpha} q_{i L}^{\alpha} \bar{q}_{j R}^{\beta} q_{i L}^{\beta}, \quad Q_{3}^{q_{i} q_{j}}=\bar{q}_{j R}^{\alpha} q_{i L}^{\beta} \bar{q}_{j R}^{\beta} q_{i L}^{\alpha}, \\
Q_{4}^{q_{i} q_{j}}=\bar{q}_{j R}^{\alpha} q_{i L}^{\alpha} \bar{q}_{j L}^{\beta} q_{i R}^{\beta}, \quad Q_{5}^{q_{i} q_{j}}=\bar{q}_{j R}^{\alpha} q_{i L}^{\beta} \bar{q}_{j L}^{\beta} q_{i R}^{\alpha} .
\end{gathered}
$$

Here $q_{R, L}=P_{R, L} q$, with $P_{R, L}=\left(1 \pm \gamma_{5}\right) / 2$, and $\alpha$ and $\beta$ are colour indices. The operators $\tilde{Q}_{1,2,3}^{q_{i} q_{j}}$ are obtained from the $Q_{1,2,3}^{q_{i} q_{j}}$ by the exchange $L \leftrightarrow R$. The $C_{i}(\Lambda)$ are obtained by integrating out all new particles simultaneously at the NP scale $\Lambda$.

We give here as example the analytic formula for the contribution to the $B_{q}-\bar{B}_{q}$ mixing amplitudes induced by a given NP scale coefficient $C_{i}(\Lambda)$, denoted by $\left\langle\bar{B}_{q}\left|\mathscr{H}_{\text {eff }}\right| B_{q}\right\rangle_{i}$, as a function of $\alpha_{s}(\Lambda)$ :

$$
\left\langle\bar{B}_{q}\left|\mathscr{H}_{\mathrm{eff}}^{\Delta B=2}\right| B_{q}\right\rangle_{i}=\sum_{j=1}^{5} \sum_{r=1}^{5}\left(b_{j}^{(r, i)}+\eta c_{j}^{(r, i)}\right) \eta^{a_{j}} C_{i}(\Lambda)\left\langle\bar{B}_{q}\left|Q_{r}^{b q}\right| B_{q}\right\rangle,
$$

where $\eta=\alpha_{s}(\Lambda) / \alpha_{s}\left(m_{t}\right)$, the magic numbers $a_{j}, b_{j}^{(r, i)}$ and $c_{j}^{(r, i)}$ and the matrix elements can be found in eqs. (10) and (12) of ref. [12] respectively. To obtain the p.d.f. for the Wilson coefficients 

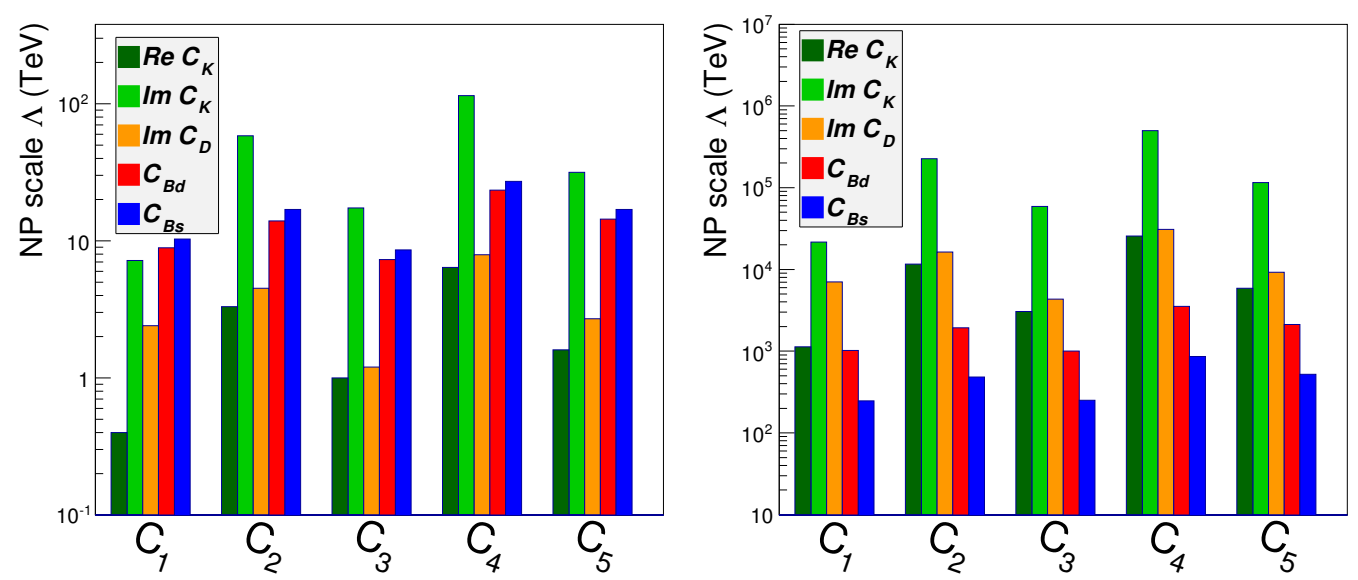

Figure 4: Summary of the 95\% probability lower bound on the NP scale $\Lambda$ for strongly-interacting NP in the NMFV scenario (left) and in the general NP scenario (right). Results from all the neutral meson systems are shown.

at the NP scale $\Lambda$, we switch on one coefficient at a time in each sector and calculate its value from the result of the NP analysis presented in sec. 2.

As we discussed in eq. (3.1), the connection between the $C_{i}(\Lambda)$ and the NP scale $\Lambda$ depends on the general properties of the NP model, and in particular on the flavour structure of the $F_{i}$. Assuming strongly interacting new particles, we have from eq. (3.1) with $L_{i}=1$

$$
\Lambda=\sqrt{\frac{F_{i}}{C_{i}}} .
$$

In the case of NMFV, we have $\left|F_{i}\right|=F_{\mathrm{SM}}$ with an arbitrary phase [11]. This condition is realised in models in which right-handed currents also contribute to FCNC processes, but with the same hierarchical structure in the mixing angles as in the SM left-handed currents.

The left plot in Fig. 4 shows the lower bounds on $\Lambda$ in a NMFV scenario, assuming strongly interacting and/or tree-level NP contributions. To obtain the lower bound on $\Lambda$ for loop-mediated contributions, one simply multiplies the bounds we quote in the following by $\alpha_{s}(\Lambda) \sim 0.1$ or by $\alpha_{W} \sim 0.03$.

We see that in the $K^{0}$ sector all bounds from non-standard operators are one order of magnitude stronger than the bound from the SM operator, due to the chiral enhancement. In addition, operator $Q_{4}$ has the strongest Renormalisation Group (RG) enhancement. In the $D^{0}, B_{d}$ and $B_{s}$ sectors, the chiral enhancement is absent, but the RG enhancement is still effective. The flavour structure of NMFV models implies that the bounds from the three sectors are all comparable, the strongest one being obtained from $\operatorname{Im} C_{K}^{4}$ (barring, as always, accidental cancellations):

$$
\Lambda_{\mathrm{NMFV}}>114 \mathrm{TeV} \text {. }
$$

In the current scenario, the $B_{s}$ system also provides very stringent constraints, especially if no new chiral structures are present.

For arbitrary NP flavour structures, we expect $\left|F_{i}\right| \sim 1$ with arbitrary phase. In this case, the constraints on the NP scale are much tighter due to the absence of the CKM suppression in the NP contributions. 
The right plot in Fig. 4 shows the results for the lower bounds on $\Lambda$ coming from all the $C_{i}$ 's for all the sectors in the case of the general NP scenario, with arbitrary NP flavour structures $\left(\left|F_{i}\right| \sim 1\right)$ with arbitrary phase and $L_{i}=1$ corresponding to strongly-interacting and/or tree-level NP. In this case the SM-like suppression of FCNC is completely absent, so that the sensitivity to NP is maximal in the $K^{0}$ sector, where the SM suppression is most effective. It remains true that the strongest bound in each sector comes from $C_{M}^{4}$. The overall constraint on the NP scale $\Lambda$ comes from $\operatorname{Im} C_{K}^{4}$ and reads

$$
\Lambda>5.0 \cdot 10^{5} \mathrm{TeV} .
$$

In conclusion, regarding the possibility of direct detection of NP at LHC, given the bounds we obtained. Clearly, a loop suppression is needed in all scenarios to obtain NP scales that can be reached at the LHC. For NMFV models, an $\alpha_{W}$ loop suppression might not be sufficient, since the resulting NP scale is $11 \mathrm{TeV}$. The general model is out of reach even for $\alpha_{W}$ (or stronger) loop suppression. Finally, the reader should keep in mind the possibility of accidental cancellations among the contribution of different operators, which might weaken the bounds we obtained.

\section{References}

[1] N. Cabibbo, "Unitary Symmetry and Leptonic Decays," Phys. Rev. Lett., vol. 10, pp. 531-533, Jun 1963.

[2] M. Kobayashi and T. Maskawa, "CP Violation in the Renormalizable Theory of Weak Interaction," Prog.Theor.Phys., vol. 49, pp. 652-657, 1973.

[3] M. Ciuchini, G. D’Agostini, E. Franco, V. Lubicz, G. Martinelli, et al., "2000 CKM triangle analysis: A Critical review with updated experimental inputs and theoretical parameters," JHEP, vol. 0107, p. 013, 2001.

[4] M. Bona et al., "The 2004 UTfit collaboration report on the status of the unitarity triangle in the standard model," JHEP, vol. 0507, p. 028, 2005.

[5] M. Bona (on behalf of the UTfit Collaboration), "Unitarity Triangle analysis in the Standard Model from the UTfit collaboration," to be found in the Proceedings of this Conference, 2016.

[6] http://www.utfit.org/.

[7] V. M. Abazov et al., "Measurement of the anomalous like-sign dimuon charge asymmetry with $9 \mathrm{fb}^{-1}$ of p p collisions," Phys.Rev., vol. D84, p. 052007, 2011.

[8] Y. Amhis et al., "Averages of b-hadron, c-hadron, and $\tau$-lepton properties as of summer 2014," 2014.

[9] R. Aaij et al., "Measurement of the CP asymmetry in $B_{s}^{0}-\bar{B}_{s}^{0}$ mixing," Phys. Rev. Lett., vol. 117 , no. 6, p. 061803, 2016.

[10] M. Bona et al., "Model-independent constraints on $\Delta F=2$ operators and the scale of new physics," JHEP, vol. 0803, p. 049, 2008.

[11] K. Agashe et al., "Next to minimal flavor violation," hep-ph/0509117, 2005.

[12] D. Becirevic, M. Ciuchini, E. Franco, V. Gimenez, G. Martinelli, A. Masiero, M. Papinutto, J. Reyes, and L. Silvestrini, " $B_{d}-\bar{B}_{d}$ mixing and the $B_{d} \rightarrow J / \psi K_{s}$ asymmetry in general SUSY models," Nucl. Phys., vol. B634, pp. 105-119, 2002. 